\title{
IL “CAVALIERE DELL'OCA” CACCIATO DALLA SCALA. IL FIASCO MILANESE DEL LOHENGRIN (1873) E IL SUO CONTESTO
}

\author{
EMILIO SALA (*)
}

Al rispettabile pubblico del caffè Gnocchi della Galleria nuova fu ammanito [sic] dai quattro musicisti del concerto invisibile, un prelibato manicaretto. Nientemeno che il preludio (Filippi direbbe ouverture) del primo atto del Lohengrin. Ma tra il moca e il gelato, tra la birra e l'acqua seltz, quel preludio invece di piacere, come al pubblico di Bologna..., fu spietatamente zittito.

«Lo Spirito folletto» (14 dicembre 1871)

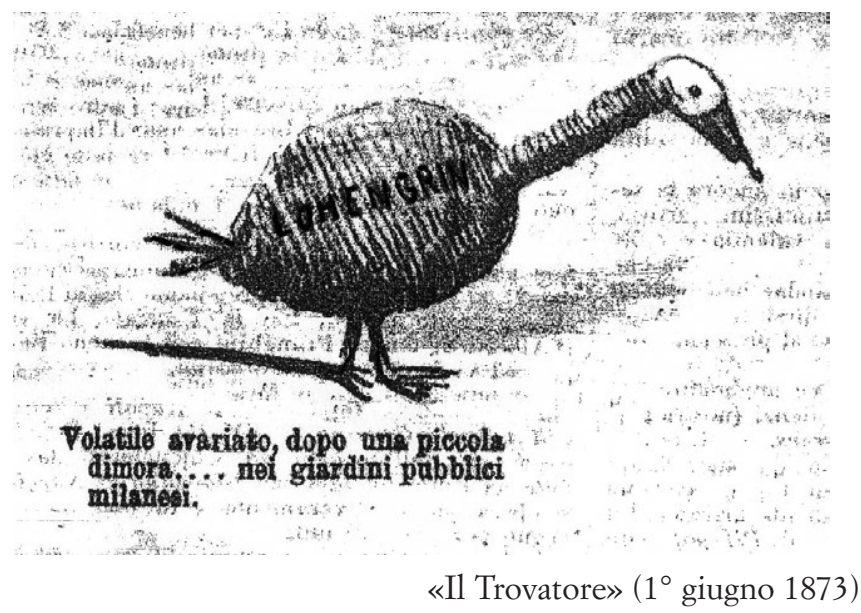

(*) Università degli Studi di Milano, Direttore scientifico dell'Istituto Nazionale di Studi Verdiani. 
RiAsSUNTO - A Milano l'opposizione Verdi vs Wagner diventò una costruzione culturale ben definita nel periodo che va dal trionfo della prima italiana del Lohengrin (Bologna, 1871) al terribile fiasco che la stessa opera incontrò a Milano (1873). Attraverso l'analisi di nuove fonti (anche iconografiche), tratte soprattutto dalla stampa umoristica dell'epoca, il mio lavoro si è posto l'obiettivo di ricostruire il contesto discorsivo che ha dato forma al rifiuto milanese del Lohengrin. Tale lavoro, di stampo archeologico-genealogico, parte dal presupposto che i fenomeni musicali sono inseparabili dai discorsi (nel senso di Foucault) che di volta in volta li inquadrano e li sostanziano. Un altro aspetto fondamentale che ho preso in esame è quello delle complesse dinamiche identitarie che caratterizzano la cultura milanese all'indomani dell'Unità d'Italia. Per citare Axel Körner, che si riferisce soprattutto alle città di Parma e di Bologna, «the cities' cultural representation speaks a local language as well as national and transnational language». Questo vale, a maggior ragione, anche per il caso di Milano.

ABSTRACT - In Milan the opposition Verdi vs Wagner became a well-defined cultural construction in the period starting with the triumph of Lohengrin Italian première (Bologna, 1871) up to the clamorous fiasco the opera received in Milan (1873). Through an analysis of new sources (also iconographical), mostly taken from the contemporary humor periodicals, this article aims to reconstruct the discursive context that shaped Milan rejection of Lobengrin. The premise for this archeo-genealogical investigation is that musical events cannot be separated from the discourses (in a Foucault-like sense) that frame and feed them. Another fundamental aspect that must be considered is the complexity of identity dynamics which characterizes Milan culture soon after Italian unification. As Axel Körner affirms, referring to Parma and Bologna, «the cities' cultural representation speaks a local language as well as national and transnational language». This is true, even more, for Milan.

Immagino di non essere stato il solo a provare un certo disagio di fronte alle anacronistiche polemiche che hanno accompagnato l'apertura della stagione scaligera di quest'anno (stagione 2012-2013): molti commentatori hanno considerato come discutibile e per certi versi riprovevole l'aver inaugurato l'anno del bicentenario di Verdi e Wagner con un'opera del secondo invece che del primo. Mutatis mutandis, sembra che alcuni atteggiamenti emersi centoquaranta anni fa durante la famosa e tumultuosa première scaligera del Lohengrin non siano ancora del tutto evaporati. Consegnando alla città di Milano i manoscritti verdiani acquistati da Eni e Intesa Sanpaolo all'asta londinese di Sotheby's, Paolo Scaroni (amministratore delegato di Eni) ha dichiarato coram populo: «Lo considero un atto riparatorio, un parziale risarcimento al fatto che la Scala abbia aperto con Wagner nell'anno del bicentenario di Verdi. La nostra più importante istituzione musicale, il Teatro alla 
Scala, deve celebrare in maniera più attenta la straordinaria storia lirica italiana. E sono sicuro che il sindaco Giuliano Pisapia concorderà con me sulla sede naturale di esposizione dei documenti: il Museo Teatrale alla Scala». ${ }^{1}$ Non credo - naturalmente - che sia possibile capire la "straordinaria storia lirica italiana" della seconda metà dell'Ottocento senza Wagner ma forse si possono comprendere meglio le polemiche dei mesi scorsi, al di là delle strumentalizzazioni legate alle contingenze del presente, se si ritorna a considerare il contesto in cui ebbe luogo la "prima" milanese del Lohengrin centoquaranta anni fa.

\section{VERDI CONTRO WAGNER}

Nel settimanale umoristico edito da Sonzogno e intitolato «Lo Spirito folletto» venne così commentata la settima e ultima rappresentazione del Lohengrin alla Scala di Milano (30 marzo 1873) che venne interrotta dai fischi alla fine del secondo atto.

«Dopo aver tranguggiato [sic] per sei lunghe, eterne sere lo spettacolo della musica dell'avvenire alla Scala nella speranza di essere presenti al felice momento che sarebbe stata seppellita per sempre, fu un dolce conforto lo spettacolo di domenica quando quella speranza divenne realtà. [...] Wagner ha fatto la fine del suo eroe dell'oca. [...] La stessa sera che qui cadeva il cavaliere dell'oca, al San Carlo di Napoli succedeva un'eruzione di applausi all'Aida di Verdi. A quanto scrivono non s'ebbe mai esempio di tanto entusiasmo. Un giornale avvenirista di Milano a questa notizia che forse gli fa rabbia, esclamò: qual differenza tra la Scala e il San Carlo. Che asino! (scusa se la mi è scappata) non vuoi che ci sia differenza tra un'opera bella ed un aborto?». ${ }^{2}$

Il «giornale avvenirista» preso di mira è probabilmente «Il Pungolo», il cui critico musicale (Leone Fortis) firmava le sue appendici con l'impegnativo pseudonimo di Dottor Verità. ${ }^{3}$ Ma il capofila degli

1 La dichiarazione è stata pubblicata (tra l'altro) nel quotidiano «La Stampa» del 20 dicembre 2012.

2 «Lo Spirito folletto» (3 aprile 1873); articolo pubblicato nella sezione I teatri e firmato $\ll$ R.».

3 Sul «Pungolo» (31 marzo 1873) si segnala l'esito trionfale dell' Aida a Napoli nello stesso giorno in cui a Milano cadeva definitivamente il Lohengrin (cioè il 30 
avveniristi a Milano era il critico musicale della «Perseveranza», quel Filippo Filippi che fin dal 1870 - come vedremo - si era battuto perché il Lohengrin fosse rappresentato in Italia. Milano era stata la terza città italiana a proporre l'opera di Wagner, ma nella «capitale morale», a differenza di quanto era accaduto a Bologna e a Firenze, il Lohengrin venne decisamente rifiutato. Dopo le prime due turbolente e fischiatissime rappresentazioni del 20 e 21 marzo («Il Pungolo» arrivò a parlare a questo proposito di «guerra civile musicale»), l'impresa cercò di calmare gli animi rispolverando il 22 marzo (giorno della commemorazione delle Cinque giornate) Un ballo in maschera di Giuseppe Verdi. Ecco come venne recensita la serata "riparatrice" sulla «Gazzetta di Milano»:

«Ieri sera c'era molta gente al teatro alla Scala per assistere alla rappresentazione del Ballo in maschera di Verdi. Il teatro non pareva più quello della sera precedente. C'era vita. Si vedeva che il pubblico sentiva quelle emozioni di cui va in traccia in teatro. Le note nervose, inspirate della musica di Verdi lo trasportavano: e gli applausi piovevano caldi, appassionati, frequenti. Il vasto recinto della Scala echeggiò varie volte del grido: Viva Verdi. Era una protesta contro innovazioni pericolose: era una protesta in favore della conservazione della nostra tradizione musicale». ${ }^{4}$

Lo scopo delle due citazioni proposte non è però (ancora) quello di affrontare la questione della burrascosa ricezione del Lohengrin a Milano nel 1873, su cui diremo qualcosa tra poco, bensì di mettere in evidenza il riferimento quasi automatico allo schema oppositivo "Verdi versus Wagner" che proprio allora incominciava a cristallizzarsi e che ha contribuito non poco a far sì che i due compositori, nati nello stesso anno, diventassero ben presto un esempio di "non-contemporaneità del contemporaneo". Con questa espressione Rainhart Koselleck e Carl

marzo). L'articolo finisce con queste parole: «Strana coincidenza collo spettacolo di iersera alla Scala!». Anche «La Perseveranza» ( $1^{\circ}$ aprile 1873$)$ sottolinea la concomitanza: «Mentre alla Scala si abbatteva il Lohengrin di Wagner, al San Carlo di Napoli l'Aida di Verdi andava alle stelle. È una singolare coincidenza che renderà pienamente soddisfatto l'amor proprio nazionale. [...] Questo successo è indubbiamente meritato, e deve rallegrare tutti quelli che amano l'arte nostra, ma non sarebbe stato meno splendido né meno giusto se fosse stato accompagnato a Milano da un atto di ospitale cortesia verso l'opera d'arte elevatissima d'uno straniero».

4 «Gazzetta di Milano» (23 marzo 1873); articolo anonimo, pubblicato nella sezione Teatri. 
Dahlhaus fanno riferimento alla discrasia esistente nei processi di sviluppo storico e dunque alla presa di coscienza, di fronte a molte coincidenze cronologiche, «dell'interna non-contemporaneità di quanto risulta esteriormente contemporaneo». ${ }^{5}$ Secondo Jean-Jacques Nattiez, questo sarebbe appunto il caso di Verdi e Wagner. Pur essendoci abituati a considerare dal punto di vista estetico e dell'evoluzione del linguaggio musicale il secondo posteriore al primo, dobbiamo infatti prendere atto che «Verdi e Wagner sono nati entrambi nel 1813 e la composizione del Rheingold come quella della Traviata ebbe inizio nel $1853 . . . \gg .^{6} \mathrm{Da}$ qui la critica che Nattiez muove al concetto di "generazione", specialmente quando - come avverrebbe ne Die Musik des 19. Jahrbunderts di Dahlhaus ${ }^{7}$ - le generazioni vengono considerate come una "strutture" totalizzanti e immanenti ai fenomeni del passato da indagare. In realtà come ogni schema storiografico anche quello di "generazione" è una costruzione ex post che bisognerebbe mettere al riparo da ogni tentazione ontologica e omologica (vale a dire dallo strutturalismo immanentista e generalizzante in cui «il sistema prevale sui processi $\left.\rangle^{8}\right)$. Ma deontologizzare la nozione di generazione considerandola come una costruzione dello storiografo, più che una struttura immanente ai fenomeni storici, non significa negare il suo valore euristico: i vari "discorsi" (nel senso di Foucault) con cui si è cercato di volta in volta di mettere in relazione (soprattutto oppositiva) Verdi e Wagner fanno parte del cangiante contesto o frame attraverso il quale inevitabilmente percepiamo e interpretiamo (anzi: interpretiamo e percepiamo) la loro musica. ${ }^{9}$ I fenomeni musicali sono inseparabili dalle

5 C. Dahlhaus, Grundlagen der Musikgeschichte, Köln, Gerig, 1977 (Fondamenti di storiografia musicale, trad. it. di A. M. Solmi, Fiesole, Discanto, 1980, 22). Cfr. anche R. Koselleck, Vergangene Zukunft. Zur Semantik geschichtlicher Zeiten, Frankfurt a. M., Suhrkamp, 1979 (Futuro passato. Per una semantica dei tempi storici, trad. it. di A. M. Solmi, Bologna, Clueb, 2007, 312).

6 J.-J. Nattiez, Alcuni concetti fondamentali di storiografia della musica: periodizzazione, "spirito del tempo", successione di generazioni, «Rivista di analisi e teoria musicale», 13, 1 (gennaio-giugno 2007), 7-35: 10.

7 Cfr. C. Dahlhaus, Die Musik des 19. Jabrbunderts, Wiesbaden, Akademische Verlagsgesellschaft, 1980 (La musica dell'Ottocento, trad. it. di L. Dallapiccola, Scandicci, La Nuova Italia, 1990).

8 J.-J. Nattiez, Alcuni concetti fondamentali di storiografia della musica cit., 14.

9 Sul modo in cui ho utilizzato la nozione di "contesto" (qui, nel titolo e 
formazioni discorsive che di volta in volta li inquadrano e li sostanziano. Anche lo schema generazionale è un discorso che va innanzitutto storicizzato attraverso un lavoro di tipo archeologico-genealogico. È quello che cercheremo di fare ricostruendo il contesto (o, se preferite, il "discorso") del fiasco milanese del Lohengrin.

\section{Milano contro Bologna}

Come si diceva, Milano fu la terza città ad accogliere il Lohengrin in Italia. La prima, Bologna, il $1^{\circ}$ novembre 1871 tributò all'opera di Wagner un «colossale trionfo», per citare l'entusiasmo del Filippi. ${ }^{10}$ Per citare invece lo sconforto dello «Spirito folletto», «Lipsia, Waterloo, Sedan non sono che pizzicotti in confronto di ciò che Bologna fu pei nemici della musica dell'avvenire!». La stessa produzione bolognese, diretta da Angelo Mariani, venne poi riproposta a Firenze il 9 dicembre dello stesso anno con un esito meno esaltante ma comunque caloroso. Sono tutti dati arcinoti che non sarà però inutile ribadire e nuovamente ricostruire (l'argomento è stato più volte studiato ma offre ancora parecchie zone meritevoli di approfondimento). ${ }^{11}$ Alla Scala, il 20 marzo 1873, il Lohengrin diretto da Franco Faccio fu al contrario fischiato con una violenza del tutto inusitata. Milano si oppone dunque dichiaratamente a Bologna e a Firenze. Si tratta per altro di un contrasto sentito e tematizzato sia sul versante degli "avveniristi", sia su quello degli antiwagneriani. Già il 16 giugno 1870, pochi giorni prima di partire per Weimar, Filippo Filippi scrisse nella «Perseveranza» un articolo in cui loda Firenze e Bologna, due città in cui si fa «molto per la

passim), si veda, soprattutto, R. Dilley, Introduction, in Id. (edited by), The problem of the context, Oxford (NY), Berghahan Books, 1999, 1-46.

10 Si veda l'appendice di Filippo Filippi nella «Perseveranza» del 6 novembre 1871.

11 Non è nemmeno il caso di tentare una breve rassegna bibliografica. Si veda, almeno, U. Jung, Die Rezeption der Kunst Richard Wagners in Italien, Regensburg, Bosse, 1974 e la sua non completa trad. it. (La fortuna di Wagner in Italia), in G. Rostirolla (a cura di), Wagner in Italia, Torino, ERI, 1982, 57-225. Sulla "prima" bolognese del Lohengrin, segnalo il recente contributo di M. Giani, «Lohengrin» a Bologna il $1^{\circ}$ novembre 1871, in P. Mioli (a cura di), Sonata a tre, 1867-1871: Verdi, Wagner e Bologna, Lucca, LIM, 2013, 17-40. 
diffusione della coltura musicale, assai più e meglio che a Milano, dove di musica e di teatri si parla molto oziosamente e si affetta uno sviscerato amore per la musica classica, ma tutto si riduce a romore di parole, anziché di note». Lo schema oppositivo è come si vede molto enfatizzato da Filippi. «Le due città che dovrebbero servire d'esempio a Milano per attività musicale, per copia di buoni studii, sono Firenze e Bologna». Segue un ammirato resoconto delle iniziative promosse dalla Società del quartetto fiorentina e dal Liceo musicale di Bologna. La frase finale dell'articolo è assai polemica: «A Milano nulla di tutto questo». ${ }^{12}$ Non stupisce allora che, nelle settimane precedenti la première milanese del Lohengrin, «Lo Spirito folletto», giornale umoristico meneghino già più volte citato, abbia denunciato una combutta tra Camillo Casarini, ex-sindaco di Bologna, e gli "avveniristi” guidati dal Filippi per «portar via da Milano le glorie, la fama, la storia del Teatro alla Scala, per farne un Areopago nella dotta città delle Bondiole». Riproduco alla Fig. 1 la vignetta che su questo tema venne pubblicata il 13 febbraio 1873 .

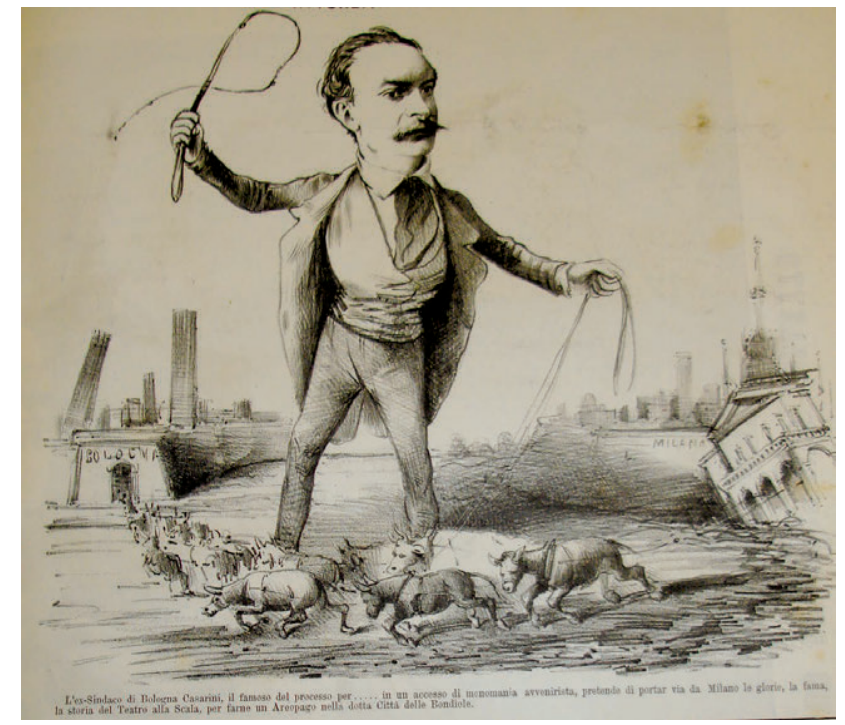

Fig. 1.

12 Le citazioni sono tratte da «La Perseveranza» del 16 giugno 1870. 
Già nel 1988, Fabrizio Della Seta aveva fatto presente che «la storia della recezione wagneriana in Italia non s'è finora sufficientemente posta il problema del ruolo svolto da Meyerbeer nel trentennio precedente la prima bolognese del Lohengrin». ${ }^{13}$ Oggi, dopo venticinque anni in cui gli studi meyerbeeriani hanno compiuto molta strada, appare chiaro che nella costruzione culturale "Milano versus Bologna e Firenze" la ricezione di Meyerbeer in Italia ha giocato un ruolo fondamentale. Non a caso a Firenze erano state rappresentate le "prime" italiane di Robert le diable, Les huguenots e Le prophète (rispettivamente nel 1840, 1841 e 1852); a Bologna Angelo Mariani aveva diretto la première italiana dell'ultima opera di Meyerbeer, L'africaine, nel 1865. Nel 1869, sempre a Bologna, Mariani, in attesa del Lohengrin, aveva proposto una sorta di festival meyerbeeriano dirigendo Robert le diable, Ugonotti e Profeta. ${ }^{14}$ In questo quadro è altamente significativo che il Mefistofele dell'avvenirista Arrigo Boito dopo essere stato fischiato a Milano nel 1868 sarebbe stato applaudito a Bologna nel 1875. Il successo del Lohengrin bolognese preparò la strada alla rivincita del nuovo Mefistofele di Boito nella "dotta città delle bondiole". D'altronde il collegamento tra il fiasco del Mefistofele e quello del Lohengrin è onnipresente nella stampa periodica milanese del 1873. E ciò avviene anche prima dell'andata in scena dell'opera di Wagner assumendo un chiaro valore prolettico: l'esperienza del fiasco del Mefistofele rafforza nel pubblico milanese l'aspettativa di una caduta del Lohengrin nella città

13 F. Della Seta, L'immagine di Meyerbeer nella critica italiana dell'Ottocento e l' $i$ dea di "dramma musicale", in M.T. Muraro (a cura di), L'opera tra Venezia e Parigi, atti del convegno internazionale di studi promosso dalla Fondazione Giorgio Cini (Venezia, 11-13 settembre 1986), Firenze, Olschki, 1988, 147-176: 167-168. Ora, con una piccola variante ( «Solo da poco tempo la storia della ricezione wagneriana in Italia si è posta il problema del ruolo svolto da Meyerbeer nel trentennio che precede la prima bolognese del Lohengrin») e con un titolo leggermente mutato (Meyerbeer nella critica italiana dell'Ottocento e l'idea di "dramma musicale"), in Id., «...non senza pazzia». Prospettive sul teatro musicale, Roma, Carocci, 2008, 171-190: 184.

14 Per un elenco completo delle opere di Meyerbeer dirette da Angelo Mariani, si veda A. Tedesco, "Queste opere eminentemente sinfoniche e spettacolose": Giacomo Meyerbeer's influence on italian opera orchestras, in N. M. Jensen - F. Piperno (edited by), The opera orchestra in 18th-and 19th-Century Europe, 2 voll., Berlin, Berliner Wissenschafts-Verlag, 2008, II (The orchestra in the theatre. Composers, works, and performance), 185-227: 225-227. 
della Scala. Per esempio, ancora nello «Spirito folletto» (6 febbraio 1873) si legge: «Nella prossima quaresima andrà in iscena il Lohengrin di Wagner, e si teme fortemente che la massa dei buongustai dello stampo vecchio non ne vogliano sapere: per lo che è quasi sicuro un fiasco alla Mefistofele». Ci troviamo insomma di fronte a un collegamento che implica una sorta di "focolaio" nel senso di Nattiez, il punto di partenza di un nesso associativo che tende ad espandersi e a diventare un vero e proprio sistema interpretativo operante anche come "sistema di influenza" ${ }^{15}$ Abbiamo già visto che in questo "discorso" si inserisce anche l'opposizione Verdi/Wagner: quando il 22 marzo 1873 (il giorno della commemorazione delle Cinque giornate) venne ripreso Un ballo in maschera, ovvero il titolo che aveva preceduto il Lohengrin nella stagione 1872-1873, il grido "Viva Verdi!" del pubblico venne subito inteso come «una protesta in favore della conservazione della nostra tradizione musicale». Ma anche l'indomani della prima recita di Un ballo in maschera (22 febbraio 1873), quando il Lohengrin era ancora abbastanza lontano, «Lo Spirito folletto» interpreta l'opera di Verdi in opposizione a quella di Wagner:

«Era voce generale, poche sere or sono, nel teatro alla Scala, che al sentire la musica del Ballo in maschera si poteva respirare a pieni polmoni. Non lo si diceva per far rabbia ai pochi avveniristi che sono tra noi; era proprio l'espressione sincera d'un sentimento comune. [...] L'impresa corre il rischio d'aver fatto un'affarone [sic] mettendo in scena quest'opera, mentre credeva che fosse un semplice mezzo per tirar avanti qualche sera. [...] Ma consoliamoci che quanto prima avremo un'altra rinfrescata ai polmoni: $i$ giornali politici ci informano che sono incominciate le prove del Lohengrin. Il Ballo in maschera è un brutto tiro al profeta dell'avvenire». ${ }^{16}$

Lo stesso vale a maggior ragione allorché, nel numero del 27 marzo 1873, «Lo Spirito folletto» pubblica un'umoristica lettera aperta a Riccardo Wagner che esordisce così: «Perdonate se vengo a disturbarvi con questa mia, che faccio tradurre in tedesco dal comune amico Boito, giacché so che voi, genio musicale, non avete ritenuto necessario studiare la lingua dell'antica patria che volete riformare». Il riferimento

15 Sulla nozione di "focolaio", vedi J.-J. Nattiez, Alcuni concetti fondamentali di storiografia della musica cit., 17.

16 «Lo Spirito folletto» (27 febbraio 1873). 
è alla famosa lettera - datata Lucerna, 7 novembre 1871 - che sull'onda del successone bolognese del Lohengrin Wagner aveva indirizzato a Boito e che quest'ultimo aveva tradotto e fatto pubblicare su «La Perseveranza» (poi ripresa anche da vari altri giornali). Orbene la lettera dello «Spirito folletto», prende in giro il carattere élitario degli "avveniristi" e associa il Lohengrin al Mesfistofele per poi contrapporlo alle ultime opere di Verdi applaudite a Milano:

$\ll[\mathrm{Non}]$ crediate che la mia ammirazione per voi sia nata nell'ascoltare il vostro Lohengrin: no, vi ammirava anche prima, senza aver inteso niente del vostro e semplicemente perché, appartenendo all'alta aristocrazia della nostra città, sono in debito di essere Wagnerista per progetto. Anche mia moglie lo è non meno, e le sere scorse non si peritò di battere le mani al Lohengrin, come le aveva battute al Mefistofele, mentre avrebbe creduto di perdere la nobiltà se avesse fatto altrettanto coll'Aida, col Don Carlos e colla Forza del destino».

Come si vede l'opposizione "Milano vs Bologna" incrocia costantemente quella "Verdi vs Wagner". Le ragioni di fondo che portarono alla sovraesposizione negativa del Lohengrin a Milano sono le stesse che condussero alla sovraesposizione positiva dei Goti di Stefano Gobatti a Bologna (per citare un altro caso emblematico, oltre a quello del Mefistofele, di dissociazione tra esperienza e aspettative tipica del nostro contesto).

Per documentare ulteriormente il processo di cristallizzazione dello schema interpretativo che siamo andati ricostruendo, vale forse la pena di citare alcuni articoli apparsi su un altro periodico musicale (settimanale) di tendenza umoristica: «Il Trovatore». Abbiamo già riportato in esergo 1'"oca-fiasco" che sintetizza la metamorfosi del cigno in un ben più modesto volatile dopo i fischi ricevuti alla Scala. Si tratta di un tema onnipresente nelle vignette giornalistiche sul quale ritorneremo ancora. Ma va detto che anche nel «Trovatore» il rifiuto di Wagner passa attraverso la milanesizzazione di Verdi. Il 10 febbraio 1872, due giorni dopo la "prima" italiana dell' Aida, «Il Trovatore» sottolinea il legame che unisce Milano e Verdi: dopo aver lanciato il giovane compositore ai tempi del Nabucco, la Scala ha allestito «l'apogeo della sua gloria, colla riproduzione del Don Carlos e della Forza del destino, cui Milano fu che ridiede vita, e giovedì sera coll'Aida». ${ }^{17}$ Per

17 «Il Trovatore» (10 febbraio 1872). 
tutte queste ragioni, «Il Trovatore» propone al Municipio che «quel Teatro che fu al grande compositore culla e campo di vittorie e di trionfi, suggellati da quello splendidissimo del suo ultimo lavoro, l'Aida, abbia a intitolarsi in suo omaggio Teatro Verdi». ${ }^{18}$ Ora, non è un caso che nello stesso numero venne pubblicata a tutta pagina un'immagine raffigurante Elsa e Lohengrin, anche se priva di ogni componente caricaturale. Le caricature non si fecero però attendere. Il 2 marzo 1872, per esempio, uscirono una serie di vignette a doppia pagina che mettono in opposizione l'Aida milanese e il Lohengrin bolognese. In un'immagine oltremodo significativa per il nostro discorso si vede il Duomo di Milano che toglie lo scettro dalle mani della Garisenda di Bologna (i milanesi «si consolano che l'Aida ha ridato alla guglia del Duomo lo scettro musicale, che da qualche tempo stava nelle mani della Garisenda di Bologna» $\left.{ }^{19}\right)$. Ancora più emblematica è la vignetta (L'Aida al Caffè Gnocchi) che riproduciamo alla Fig. 2, in cui si prende in giro il supposto "wagnerismo" dell'Aida: a sinistra, secondo coloro che bevono birra (i wagneriani), Verdi si sarebbe inchinato al cigno; a destra, secondo coloro che bevono vino (i verdiani), egli «è sempre un'aquila, la quale s'innalza al di sopra di tutti i cigni, che non sono in realtà che tante oche». ${ }^{20}$

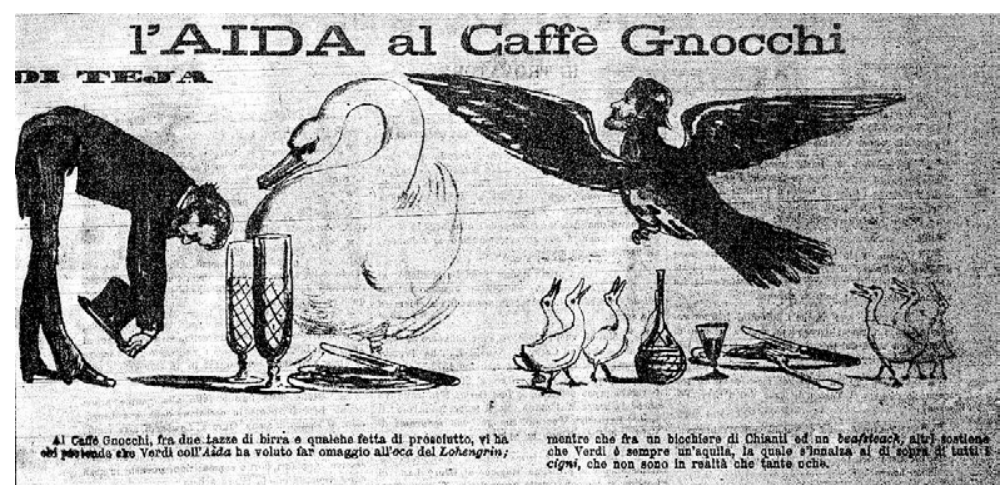

Fig. 2.
18 Ibidem.
19 «Il Trovatore» (2 marzo 1872).
20 Ibidem. 


\section{3. «VIAGGIO MUSICALE NELLE REGIONI DELL'AVVENIRE»}

Ma torniamo ai mesi che prepararono la première bolognese del Lohengrin. Negli anni 1869-1870, l'editore milanese Lucca, ottenuto il diritto esclusivo di rappresentazione delle opere di Wagner in Italia, pubblicò i primi spartiti di queste ultime, tra cui il Lohengrin. È con lo spartito pubblicato dall'editore Lucca che Verdi si recò a vedere l'opera diretta da Mariani a Bologna il 19 novembre 1871 ed è su quella riduzione per canto e pianoforte che appose le famose chiose trascritte da Alessandro Luzio nel secondo volume dei suoi Carteggi verdiani. ${ }^{21}$ D'altra parte, il 23 gennaio 1870 Verdi aveva chiesto a Camille Du Locle di spedirgli gli scritti letterari di Wagner, sottolineando che desiderava «conoscerlo anche da questo lato». ${ }^{22}$ Nell'estate dello stesso anno l'instancabile Filippi si recò a Weimar per assistere a una serie di rappresentazioni wagneriane (Der fliegende Holländer, Lohengrin, Tannbäuser e Meistersinger). Da Weimar egli pubblicò su «La Perseveranza» alcune appendici che - sotto il titolo Viaggio musicale nelle regioni dell'avvenire - ebbero un impatto fondamentale nella ricezione di Wagner in Italia. ${ }^{23}$ La partenza del «plenipotenziario italiano della musica dell'avvenire» venne fotografata su «L'Uomo di pietra» con un disegno umoristico (Fig. 3) pubblicato il 25 giugno 1870.

Il gruppo degli avveniristi che salutano il critico musicale della «Perseveranza» comprende (da sinistra a destra): Alessandro Fano (redattore del periodico «Il mondo artistico»), Leone Fortis (critico musicale del «Pungolo»), Emilio Praga, Arrigo Boito, Franco Faccio, Alberto Mazzuccato e il pittore Carlo Mancini. Filippi è rappresentato (massonicamente?) con il triangolo e il compasso, simboli della cerebrale "musica dell'avvenire". Dopo la bocciatura del Lohengrin a Milano, nello «Spirito folletto» del 27 marzo 1873 venne pubblicata questa frecciata: «Alcuni sostengono che la musica di Wagner è musica (!) studiata, matematica, aritmetica, logaritmica! È perciò dunque che

${ }^{21}$ Cfr. A. Luzio (a cura di), Carteggi Verdiani, 4 voll. (1935-1947), Roma, Accademia Nazionale dei Lincei («Studi e Documenti», 4), 1935, II, 217-220.

22 F. Abbiati, Giuseppe Verdi, 4 voll., Milano, Ricordi («Le vite»), 1959, III, 328.

23 F. Filippi, Viaggio musicale nelle regioni dell'avvenire, «La Perseveranza» (25, 28, 29, 30 giugno, 2, 4, 8 e 9 luglio 1870); articolo successivamente raccolto in Id., Musica e musicisti. Critiche, biografie ed escursioni, Milano, Brigola, 1876, 208-318. 
ha bisogno di tante prove e controprove». Non c'è spazio per un'analisi degli interessanti articoli del Filippi, ma è significativo che alla fine della recensione del Lohengrin si faccia riferimento alla questione della rappresentabilità delle opere di Wagner in Italia: «Io credo fermamente scrive il Filippi - che il Vascello fantasma, ed il Lohengrin specialmente, sieno rappresentabilissimi in Italia, e che possano piacere al nostro pubblico come piacciono a codesto, ma con un'esecuzione eguale, così accurata e così perfetta». ${ }^{24}$

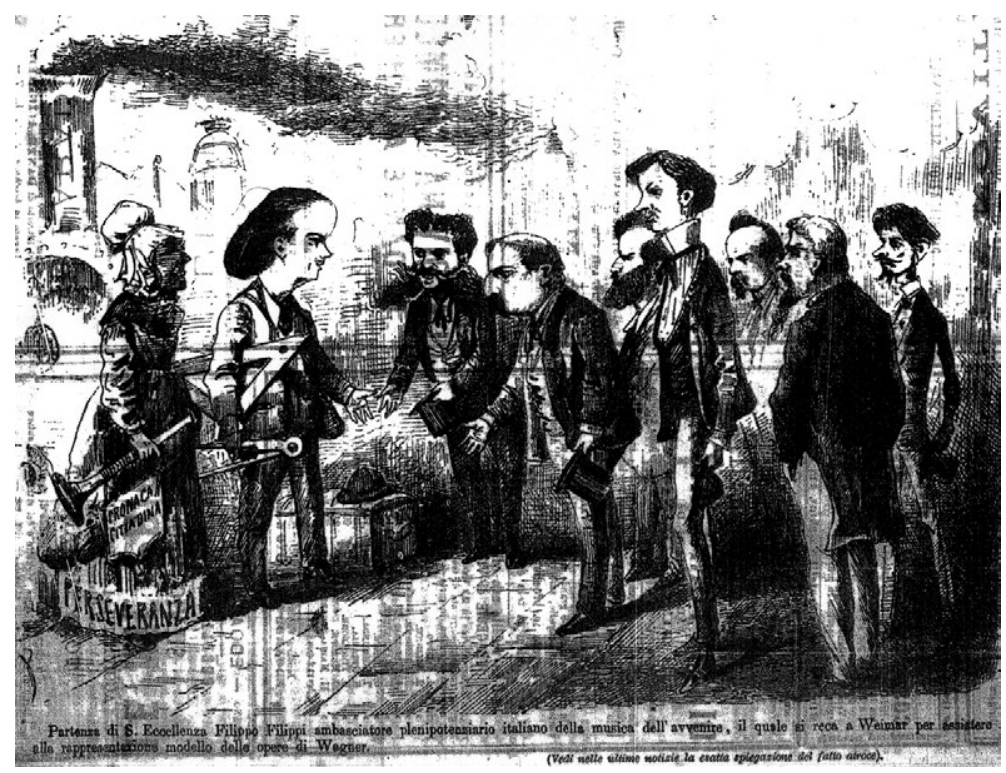

Fig. 3.

L'importanza degli articoli di Filippi è testimoniata anche dalle reazioni che seppero suscitare, e non solo nel campo avverso degli antiwagneriani per principio. A partire dal 9 ottobre 1870, infatti, Alberto Mazzuccato, lo storico collaboratore della «Gazzetta musicale di Milano», che era stato per l'introduzione di Meyerbeer in Italia ciò che Filippi stava diventando per quella di Wagner, pubblicò sulla rivista di Ricordi una serie di articoli che si riallacciano direttamente

24 «La Perseveranza» (30 giugno 1870); cit. in F. Filippi, Musica e musicisti cit., 262. 
al Viaggio musicale nelle regioni dell'avvenire uscito qualche mese prima sulla «Perseveranza». ${ }^{25} \mathrm{Il}$ contesto degli articoli di Mazzuccato è interessante anche perché rinvia alle lezioni di estetica tenute da quest'ultimo presso il conservatorio di Milano. Non a caso egli si rivolge a uno dei suoi allievi, Ruggero Laboccetta, che durante le lezioni aveva chiesto l'opinione di Mazzuccato sulle appendici del Filippi pubblicate dalla «Perseveranza». Nonostante sia collocato nel gruppo degli "avveniristi" nel disegno dell'«Uomo di pietra», Mazzuccato prende le distanze da Filippi di cui lamenta un eccesso di partigianeria. Le sue argomentazioni sembrano tuttavia un po' capziose e orientate a dare un colpo al cerchio e uno alla botte. Da una parte egli nega, con un sottinteso nazionalistico, che in Italia non si sia in grado di rappresentare e di fruire le opere di Wagner, dall'altra stigmatizza la tendenza antimelodica e anticanora insita nella drammaturgia musicale wagneriana i cui personaggi «sono tuffati in un mare di armonie sinistre, di ritmi indeterminati, opprimenti, labirintici (se mi passate il vocabolo) dove la luce, la calma, il riposo, l'uscita, s'invocano, - ma che... non vengono... non vengono mai». ${ }^{26}$ Alla fine Mazzuccato si ribella all'idea di un passaggio di testimone da Meyerbeer a Wagner. Di fronte alla predizione di Filippi che ritiene «probabile di vedere non tardi il Roberto [il diavolo] e gli Ugonotti sorpassati, nel gusto del pubblico, dal Lohengrin e dal Vascello fantasma», egli sbotta: «Oh! no, no! Questo non avverrà; né presto, né tardi, né mai». ${ }^{27}$ Comunque va anche ricordato che Mazzuccato scrive sulla rivista dei Ricordi, acerrimi nemici dell'editore Lucca. Di lì a qualche mese, Iridio Licurgo (alias Giulio Ricordi) avrebbe recensito sulla «Gazzetta musicale di Milano» il Lohengrin di Bologna riprendendo molti degli argomenti antiwagneriani di Mazzuccato e preannunciando curiosamente quello che sarebbe avvenuto il 30 marzo 1873 alla Scala:

25 A. Mazzuccato, Una lezione di filosofia musicale in ottobre. Lettera a Ruggero Laboccetta, «Gazzetta musicale di Milano» (9, 23, 30 ottobre, 13, 27 novembre, 11, 18 dicembre 1870; poi, mutato il titolo in Wagner e la melodia, 15, 22 gennaio, 26 febbraio e 12 marzo 1871).

26 Id., Wagner e la melodia, «Gazzetta musicale di Milano» (26 febbraio 1871).

27 F. Filippi, Viaggio musicale nelle regioni dell'avvenire, «La Perseveranza» (25 giugno 1870) e A. Mazzuccato, Wagner e la melodia cit. (12 marzo 1871). 
«[...] nessuno in buona fede potrà negare che, se invece del nome di Wagner circondato dalla mistica aureola dell'apostolato, il Lohengrin tale e quale si udì a Bologna avesse portato il nome di un maestro sconosciuto [tipo Boito al tempo del Mefistofele], o di un giovane principiante, sarebbe irremissibilmente caduto al secondo atto, per non rialzarsi mai più, non ostante le impressioni favorevoli lasciate dal primo». ${ }^{28}$

\section{Cosmopolitismo, IDENTITÀ NAZIONALE, IDENTITÀ LOCALE}

Bisognerà ora mettere in relazione fra loro queste tre nozioni che agiscono nel nostro contesto in una serie di combinazioni alquanto varie e contraddittorie. L'esistenza di un «canone risorgimentale» (mirabilmente analizzato da Alberto Banti) ${ }^{29}$ e l'affermazione del nazionalismo unificante non sono infatti in contrasto, specialmente dopo l'Unità, con il rafforzamento di specificità locali spesso fortemente differenziate e polemicamente dissenzienti. In questa prospettiva, può essere interessante ricorrere al concetto di «intimità culturale» elaborato da Michael Herzfeld per esprimere il distacco tra lo stato e la cittadinanza. Egli parla a questo proposito di disemia che definisce come «la tensione formale o codificata tra la presentazione ufficiale di sé e quello che accade nel privato dell'introspezione collettiva». ${ }^{30}$ I tre livelli di appartenenza (locale, nazionale e transnazionale) interagiscono continuamente fra loro in una serie di dinamiche dal carattere appunto disemico e ambivalente, ritagliando uno spazio instabile che implica - per dirla con Julian Budden - «un problema di identità». Con quest'ultima espressione, Budden intitola un importante capitolo del suo libro su Verdi dedicato alla storia dell'opera italiana dal 1870 al 1890. Il dilemma che tormenta i compositori italiani di questo periodo è innanzitutto identitario e si può riassumere così: «come fare qualcosa di nuovo senza dare l'impressione di adornarsi di penne altrui».3. Un altro riferimento bibliografico importante, in cui i tre livelli evidenzia-

28 I. Licurgo, Lohengrin di Riccardo Wagner al Teatro Comunale di Bologna, «Gazzetta musicale di Milano» (5 novembre 1871).

29 A.M. Banti, La nazione del Risorgimento. Parentela, santità e onore alle origini dell'Italia unita, Torino, Einaudi, 2000.

30 M. Herzfeld, Cultural intimacy. Social poetics in the nation-state, London, Routledge, 1997 (Intimità culturale. Antropologia e nazionalismo, trad. it. di E. Nicolcencov, Napoli, L'ancora del Mediterraneo, 2003, 29).

31 J. Budden, The operas of Verdi, 3 voll., London, Cassel, 1973 (Le opere di Verdi, a cura di G. Pestelli, 3 voll., Torino, EDT, 1988, III, 307). 
ti vengono fatti interagire costantemente e intelligentemente, è il recente libro di Axel Körner intitolato Politics of Culture in Liberal Italy (2009). Non si può che concordare pienamente con lui quando scrive che

«[...] the cities' cultural representation speaks a local language as well as national and transnational language. This last point is particulary important when refering to Italy, a country often associated with the combination of an underdeveloped political culture and agressive nationalism which paved the way for Fascism. However, when examining Italian identity, not only do we have to take account of regional diversity and strong municipal traditions, but also we should appreciate that Italian culture engages closely with wider European experiences».23

Una combinazione assai rivelatrice dei tre livelli identitari (locale, nazionale e transnazionale) è già nel "nom de plume" di Giulio Ricordi compositore. Egli firmava infatti i suoi lavori con lo pseudonimo di Jules Burgmein. Nome francese, cognome tedesco, ma con un significato che potremmo tradurre, un po' liberamente, Giulio "del suo paese". D'altra parte, la componente nazionalista non è certo assente sul versante bolognese, quello del wagnerismo cosmopolita. Quando l'editore Lucca ventilò l'ipotesi di far eseguire il Lohengrin bolognese da artisti tedeschi invece che italiani, le autorità cittadine si opposero fermamente. Ecco come Angelo Mariani narra l'accaduto a Carlo Del Signore (lettera datata Bologna, 30 luglio 1871):

«Sullo spettacolo del prossimo autunno nulla fu ancora deciso. Il Sindaco e la Giunta Municipale aveva in pensiero di fare eseguire il Lohengrin, ma avendo Lucca messo in campo delle pretese inaccettabili, credo che non se ne farà nulla. Figurati un poco! Fra le altre cose Lucca voleva che fosse venuto Bülof [Hans von Bülow] per mio consigliere, e che tutti gli artisti fossero stati tedeschi e che avessero già eseguito il Lohengrin con successo in altri teatri di Germania sotto la direzione di Wagner stesso. Questa pretesa del Lucca indignò il Sindaco e la Giunta e l'intera popolazione, e ne fu fatta quistione di decoro nazionale. Il Municipio si comportò veramente con una fermezza esemplare. Diede la più ampia soddisfazione a me e agli artisti italiani, dicendo che piuttosto preferisce tener chiuso il teatro comunale che di permettere che si sia fatta questa ingiuria al decoro musicale di Bologna e dell'Italia intera».33

32 A. Körner, Politics of culture in liberal Italy. From unification to fascism, New York-London, Routledge, 2009, 2.

33 U. Zoppi, Angelo Mariani, Giuseppe Verdi e Teresa Stolz in un carteggio inedito, Milano, Garzanti, 1947, 254. 
Tornando a Milano, gli scapigliati importano vari prodotti della modernità parigina, tra cui il nuovo vaudeville alla maniera di Eugène Labiche, ma lo riformulano in dialetto milanese: si veda La cagnotte (1864) che diventa, adattata da Cletto Arrighi, El barchett de Boffalora (1870), con le parti musicali composte da vari autori (Angelo Pettenghi, Cesare Casiraghi ed Ester Trezzini-Bignami). Insomma, un'analisi culturale del contesto in cui ci muoviamo implica sempre la compresenza dei tre livelli che abbiamo cercato di evidenziare e che sarebbe gravemente riduttivo trattare singolarmente.

Il "problema di identità" è un problema culturale che assume in quegli anni una rilevanza politica evidente: la costruzione di una retorica nazionale, nella quale Verdi gioca un ruolo fondamentale, adombra nello stesso tempo la fragilità dei nuovi valori identitari. Non è un caso che, il giorno dopo l'ultima disastrosa rappresentazione del Lohengrin a Milano, Filippi pubblichi sulla «Perseveranza» due articoli intitolati La musica alla camera dei deputati in cui commenta alcune «importanti discussioni sull'arte musicale, ch'ebbero luogo alla nostra Camera dei deputati, nelle due tornate del 31 gennajo e del 6 febbraio di quest'anno [1873]». ${ }^{34}$ Sebbene siano passati quasi due mesi da quel dibattito parlamentare, Filippi sente il bisogno di parlarne il giorno dopo la definitiva caduta del Lohengrin a Milano. Perché? Perché può così controbattere all'on. Augusto Righi, un liberale di destra che aveva evocato il pericolo della perdita del «tipo musicale italiano» per colpa - anche - di una certa critica deleteria, additando appunto il caso del Lohengrin milanese. Cosa hanno potuto suscitare gli articoli filowagneriani di Filippi presso le masse «schiave delle abitudini e dei pregiudizi» se non «uno spirito ostinato e turbolento di contraddizione»? «Non ho bisogno di appoggiare il mio asserto coi fatti: gli esempi sono recentissimi e, come si suol dire, palpitanti di attualità». ${ }^{35} \mathrm{Al}$ conservatorismo dell'on. Righi, Filippi contrappone sposandola - la posizione dell'ex sindaco di Bologna Casarini che così si rivolge al suo collega preservatore dell'identità musicale italiana:

«"Pretendereste forse che i nostri giovani, mentre gli stranieri ingigantiscono, abbiano poi a scendere a serie battaglie nell'ignoranza completa dell'immenso

34 «La Perseveranza» (31 marzo 1873).
35 Ibidem. 
movimento artistico che si svolge attorno a noi?". Il discorso del Casarini si riassume in una formola bellissima [...]: "Siamo cauti, ma siamo progressivi" ».

\section{Elsa e MARgherita}

Vorrei ora proporre una breve digressione dedicata a un'associazione musicale particolarmente diffusa nella stampa periodica milanese dei primi anni '70 - quella tra l'apparizione notturna di Elsa sul verone (scena 2 dell'atto II del Lohengrin) e il soliloquio di Margherita nel Faust di Gounod (ultima scena dell'atto III). Per capire il senso di tale associazione, va detto che il Faust era stato molto applaudito alla Scala nel 1862 e che, ripreso regolarmente negli anni successivi, diventò il paradigma dell'opera nuova straniera (postmeyerbeeriana) accettata dal pubblico milanese. L'accostamento tra il Faust e il Lohengrin può contenere insomma un sottinteso di tipo polemico: se si ammettesse l'influenza di Wagner su Gounod, infatti, risulterebbe perlomeno incoerente rifiutare violentemente l'opera del primo portando alle stelle quella del secondo. Dunque è con una certa enfasi che Filippi mette le due scene una di fianco all'altra:

«L'apparizione di Elsa sul verone cangia il colore alla musica: son suoni dolci, mesti, sereni che accompagnano un monologo di amore; nei teneri accenti di Elsa havvi la speranza di una prossima felicità, e un innocente candore, che commuove e rapisce. All'udire questo pezzo la memoria corre subito alla scena della finestra del Faust di Gounod, quando Margherita parla alle stelle ed ai fiori del suo amore: è lo stesso stile, lo stesso linguaggio, lo stesso colorito istromentales. ${ }^{37}$

Non è un caso che durante i giorni della disfatta del Lohengrin alla Scala, Paolo Ferrari, che recensì l'opera di Wagner sul «Pungolo» al posto di Leone Fortis (in quei giorni fuori Milano), abbia sentito il bisogno di smentire l'associazione portata avanti nel campo degli avveniristi: «No signori - il Faust appartiene alla vecchia maniera: tra Faust e Lohengrin c'è il suddetto abisso». ${ }^{38}$ D'altra parte Filippi cercò anche di essere più preciso. In un resoconto del Lohengrin di Bologna, egli

\footnotetext{
36 «La Perseveranza» $\left(1^{\circ}\right.$ aprile 1873$)$.

37 Ibidem (30 giugno 1870).

38 «Il Pungolo» (24 marzo 1873); articolo firmato: «P[aolo]. F[errari].».
} 
cita un luogo specifico in cui circostanziare meglio il parallelismo proposto: «Ci sono poi degli accordi che il pubblico prese per stonazioni, mentre non lo sono punto, specialmente quello di la bemolle, do bemolle dopo la risoluzione in si bemolle della così deliziosa aria che Elsa canta alla finestra, e di cui Gounod si valse tanto nel Faust e in Romeo $e$ Giulietta». ${ }^{39}$ Non so bene dove Gounod si sarebbe valso di procedimenti come quello indicato, ma è evidente che Filippi pensa a quello che succede alla fine del Lied di Elsa che si conclude (sulle parole «in Liebe!») appunto in Si bemolle maggiore (in $p p$ ). Il bicordo La bemolle-Do bemolle (in $s f / p p$ ) che segue, intonato da due oboi e due corni, ci porta in un contesto tonale lontanissimo (Mi bemolle minore?), continuamente ondivago e cosparso di settime diminuite destabilizzanti, fino a che non si afferma il Sol minore di Ortrud ( $«$ In ferner Einsamkeit des Waldes»). Anche l'ultima parte del canto di Marguerite, dopo la cadenza in Sol bemolle minore (in $p p$ ) sulle parole «l'air m'enivre!», procede in modo continuamente cangiante e aperto, fino al coup de tamtam (in ff) che coincide con la sardonica risata di Mefistofele (cui segue la ripresa del tema in fff e in $\mathrm{Fa}$ maggiore), ma con una ricerca dell'effetto molto lontano (per noi, oggi) dall'arte della "transizione" wagneriana. Semmai le due scene si assomigliano di più all'inizio, nel colore dei legni (soprattutto nel profilo del flauto: «dolce» in Wagner, «dolcissimo» in Gounod) e nel modus canendi delle due eroine. Epperò senza la coincidenza situazionale e l'analogo contesto scenico-visivo tutto il discorso di Filippi non riuscirebbe a convincerci più che tanto. Nella nostra prospettiva, il suo interesse riguarda più il livello ricettivo (a parte subiecti) che il livello analitico (a parte obiecti). Insomma: Filippi usa Gounod per (cercare di) far accettare Wagner al pubblico scaligero.

\section{HiC IACET LOHENGRIN}

È ben nota l'importanza della caricatura e della satira nella diffusione della moda wagneriana in Europa. Nel catalogo della mostra Fortuny e Wagner. Il wagnerismo nelle arti visive in Italia è stata tra l'altro ripresa una divertente vignetta (Caravana del Lohengrin) tolta dal libro di John Grand-Carteret, Richard Wagner en caricatures. 130 repro-

39 «La Perseveranza» (6 novembre 1871). 
ductions de caricatures françaises, allemandes, italiennes, portraits, autographes (Paris, Larousse, 1892, p. 235), una vignetta che si riferisce al trasferimento del Lohengrin da Bologna a Firenze di cui abbiamo già parlato. Come si può vedere alla Fig. 4 (dove riproduciamo l'immagine), l'impresario Luigi Scalaberni, a cavallo di un cigno tirato da una locomotiva a vapore è raffigurato in viaggio verso Firenze: «il piccolo personaggio che spunta dalla gerla è il cavalier Antonelli, direttore dei concerti pubblici di Bologna e dell'orchestra del teatro, ma nello stesso tempo produttore di acqua di seltz, ch'egli spruzza dispettosamente sulla testa dell'animale». ${ }^{40} \mathrm{Dal}$ taschino della giacca dell'impresario fuoriesce il direttore Angelo Mariani in veste di mago.

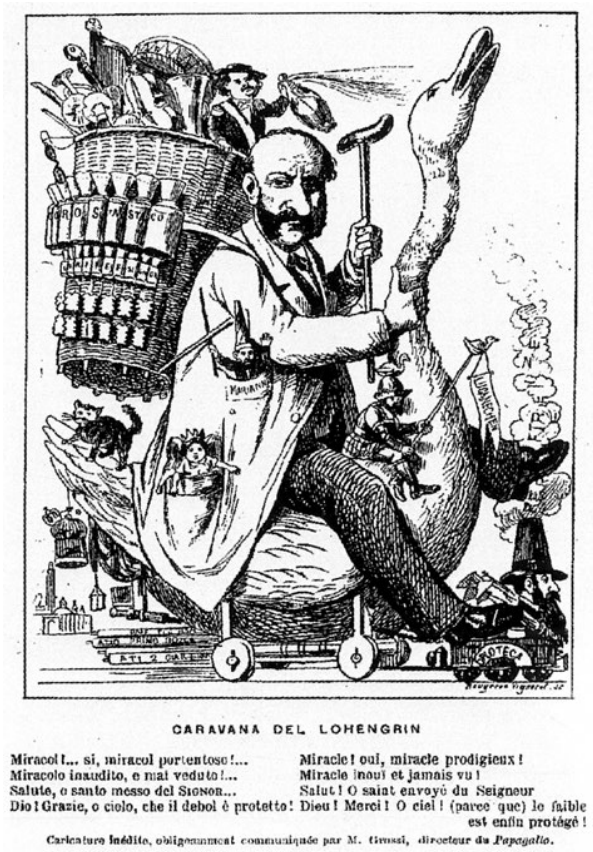

Fig. 4.

40 P. Bolpagni, Wagner nell'illustrazione e nella grafica effimera in Italia. Dal manifesto alla cartolina, dalla caricatura alla figurina Liebig, in Id. (a cura di), Fortuny e Wagner. Il wagnerismo nelle arti visive in Italia, Ginevra-Milano, Skira, 2012, 189-194: 191. L'immagine della Fig. 4 è riprodotta a p. 199 di questo catalogo. 
Sulla scia di questo esempio e degli altri due già citati vorrei segnalare ancora un paio di caricature antiwagneriane, firmate da Snebel e pubblicate sul milanese «Lo Spirito folletto» nella primavera del 1873, quando ormai il disastroso esito del Lohengrin alla Scala era un fatto compiuto. Entrambe le immagini sono riprodotte con grande enfasi e stampate a doppia pagina. Esse coincidono certamente con il punto apicale dell'antiwagnerismo meneghino e lo illustrano in modo assai eloquente. La prima si intitola Lohenfrin-frin. Piedilavoro senza avvenire (Fig. 5) e venne pubblicata il 27 marzo 1873.
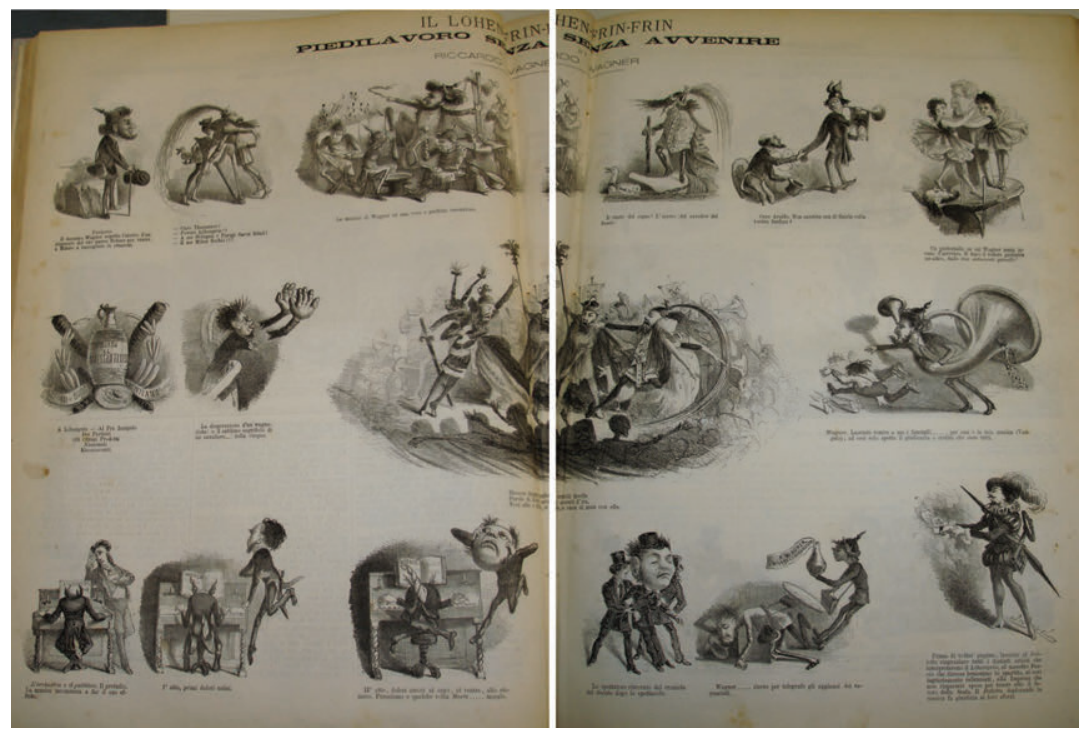

Fig. 5.

Nella prima vignetta in alto a sinistra, con tanto di elmetto prussiano in testa (ma anche con curiosi connotati giudaici), ${ }^{41} \ll i l$ maestro

41 Per quanto riguarda le (eventuali) connotazioni giudaiche presenti nell'immagine di Wagner (l'elmetto prussiano indossato quasi come una kippah, il naso adunco, la barbetta caprina all'insù e l'atteggiamento da "errante"), esse aprirebbero un capitolo tanto interessante quanto controverso che posso qui solo sfiorare. In Italia l'antisemitismo wagneriano non è un elemento particolarmente tematizzato nella stampa periodica, però può essere interessante fare riferimento al libro Die Juden in der Karikatur di Eduard Fuchs in cui si trovano molte vignette antigiudaiche paragonabili 
Wagner aspetta l'arrivo d'un dispaccio del suo amico [Gustav] Böhm per venire a Milano a raccogliere le ovazioni». Böhm era il régisseur del Teatro Nazionale di Budapest, scritturato dalla Scala per sovraintendere alla mise en scène del Lohengrin milanese. ${ }^{42}$ Naturalmente alla fine il dispaccio sarà assai poco favorevole alla causa wagneriana. $\mathrm{Ma}$ quello che conta di più mettere in evidenza è l'associazione suggerita tra la musica di Wagner e la rivelazione - dopo Sedan - della potenza prussiana. Le reazioni di Verdi alla sconfitta dei francesi sono troppo note per essere citate, ma i riferimenti al contesto politico sono diffusissimi nella stampa periodica dell'epoca. Mazzuccato inizia la serie dei suoi articoli in risposta a Filippi evocando le «immense sciagure di un popolo fratello» e nei giorni che precedettero la "prima" del Lohengrin alla Scala girò la voce «che i francesi stabiliti a Milano avevano risoluto di prendersi, a spese di Wagner, una rivincita di Sedan, e che i tedeschi difenderebbero energicamente il loro patriotta [sic], sicché il teatro alla Scala diventerebbe un campo di battaglia». ${ }^{43}$

Nella seconda caricatura (Fig. 6), pubblicata nello «Spirito folletto» del 24 aprile 1873, viene rappresentata la pietra tombale posta sopra il Lohengrin milanese, in calce alla quale si vede il popolo meneghino unito a respingere il "cavaliere dell'oca" verso la "via dell'avvenire" da dove era venuto.

Questa caricatura è con ogni probabilità una risposta alle Riflessioni postume sul Lobengrin pubblicate il 9 aprile 1873 nel «Mondo artistico» che culminano con la seguente affermazione: «Signori antiwagneriani [...] oggi avete seppellito il Lohengrin sotto i fischi: verrà il giorno in cui lo ascolterete».

a quella del compositore compresa nella Fig. 5. D'altronde lo stesso Fuchs sottolinea una certa complementarietà tra l'autore di Das Judenthum in der Musik e il mondo ebraico: «Der Antisemit Richard Wagner hat keine fanatischeren Verehrer als die Juden» (E. Fuchs, Die Juden in der Karikatur. Ein Beitrag zur Kulturgeschichte, München, Albert Langen, 1921, 264).

42 Come scrisse «Il mondo artistico» (31 marzo 1873), «La messa in iscena fa onore all'impresa. L'opera del maestro Böhm, direttore di scena del teatro di Pest, venuto a Milano per dirigere la parte, dirò così, scenografica e coreografica del Lobengrin è stata utilissima» (articolo firmato: «Ipsilon»).

${ }^{43}$ Le due citazioni sono tratte, rispettivamente, dalla «Gazzetta musicale di Milano» (9 ottobre 1870) e da «Il mondo artistico» (15 marzo 1873; articolo firmato: «Ipsilon»). 


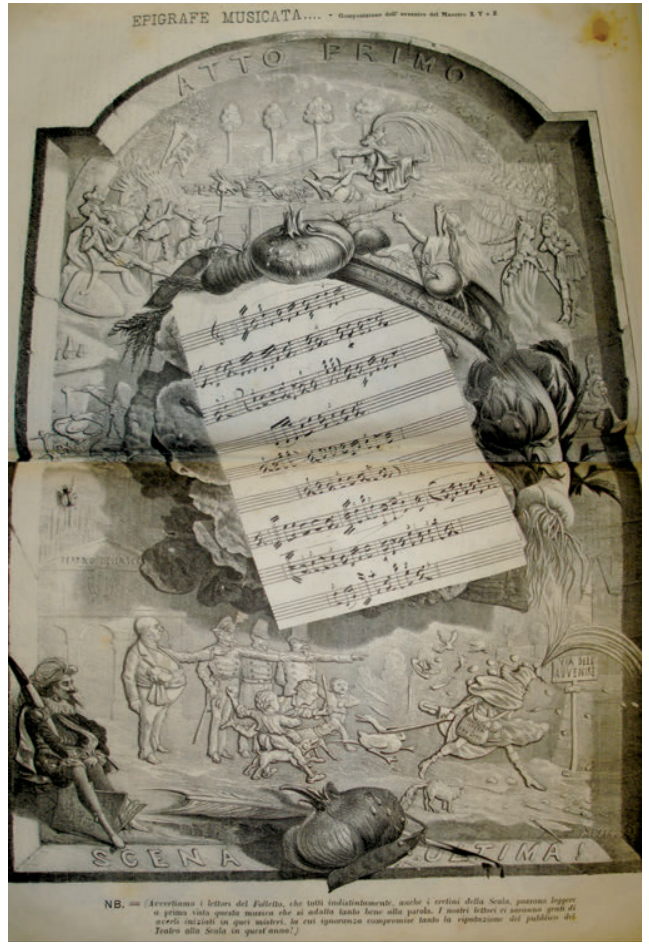

Fig. 6.

\section{CONCLUSIONI}

Nel suo saggio Difficoltà della storiografia dell'opera italiana, Della Seta ha discusso l'applicazione alla storia dell'opera dei concetti koselleckiani di "spazio di esperienza" (Erfahrungsraum) e di "orizzonte d'attesa" (Erwartungshorizont), sottolineando come debbano essere tenuti ben distinti: «l'aspettativa è predisposta dall'esperienza, ma non coincide totalmente con essa». ${ }^{44} \mathrm{Se}$, come sostiene Koselleck, la tensione tra esperienza e aspettativa aumenta progressivamente nell'età moderna, essa diventa deflagrante nel caso della ricezione di Wagner in Italia: il discorso sulla "musica dell'avvenire" implica un'aspettativa che

${ }^{44}$ F. Della Seta, Difficoltà della storiografia dell'opera italiana, in Id., «...non senza pazzia». Prospettive sul teatro musicale cit., 135-148: 146. 
tende a distaccarsi sempre più dalle esperienze precedenti. Ma ciò che più conta è la trasformazione cui viene sottoposto lo spazio di esperienza tradizionale. Non bisogna dimenticare, infatti, che «anche le esperienze si trasformano, sebbene una volta fatte siano sempre le stesse. È questa la struttura temporale dell'esperienza, che non può essere accumulata senza l'effetto retroattivo dell'aspettativa». ${ }^{45}$ Questo effetto retroattivo delle aspettative che trasformano lo spazio delle esperienze già immagazzinate appare evidente per esempio nella recensione del Lohengrin bolognese di Giulio Ricordi. Di fronte al successo dell'opera di Wagner, Ricordi pensa al fiasco milanese del Mefistofele secondo uno schema associativo onnipresente - come abbiamo visto - all'interno del nostro contesto. «Se il Lohengrin - scrive un po' provocatoriamente Ricordi - avesse portato il nome di Boito, avrebbe finito coi fischi spietati, come finì il Mefistofele». Ed è qui che scatta la rivalutazione retrospettiva di almeno una parte dell'opera di Boito, sintomo di un cambio di prospettiva circa il suo valore (già esperito come negativo) e la possibilità di una sua eventuale riabilitazione. Ricordi non ha paura di sollevare «scandali più forti ancora se dirò che in tutto il Lohengrin non si riscontra un quadro così vasto, così lungamente $\mathrm{e}$ grandiosamente svolto come il prologo del Mefistofele». ${ }^{46}$ Abbiamo già detto che a Bologna il successo del Lohengrin preparò la strada alla riabilitazione del Mefistofele, così come a Milano l'insuccesso del Mefistofele fu il presupposto di quello del Lohengrin. Possiamo allora concludere che quando nel 1881 i milanesi applaudirono la nuova versione dell'opera di Boito si crearono le condizioni perché anche l'opera di Wagner potesse essere riabilitata - il che avvenne, appunto, a partire dal 1888 (quindici anni dopo il primo, terribile fiasco).

45 R. Koselleck, Futuro passato. Per una semantica dei tempi storici cit., 308.

46 «Gazzetta musicale di Milano» (5 novembre 1871). 